\title{
Shortcomings of natural family planning methods
}

This Letter contained a few errors (BMJ 2012;345:e5566, doi:10. 1136/bmj.e5566), which were introduced during editing. Firstly, the second sentence of the penultimate paragraph should have read: "Pills, condoms, FABMs, and even depot medroxyprogesterone acetate require continuing commitment [not "Pills, condoms, FABMs, and even implants require continuing commitment"]." Secondly, the initials at the start of the competing interests statement should have been DAV [not
DAK, as published]. Finally, we should have included a link to the full rapid response

(www.bmj.com/content/345/bmj.e4908/rr/596880) at the end of the Letter.

Cite this as: BMJ 2012;345:e5994

๑ BMJ Publishing Group Ltd 2012 